\section{DENTRO DE LA ESCUELA, CON LA ESCUELA ADENTRO: INDAGANDO EN EL PROCESO DE LA PROPIA SOCIALIZACIÓN SOBRE EL ACCESO AL COLEGIO Y EL CUERPO EN EL AULA A TRAVÉS DE UN EJERCICIO DE TRIO-ETNOGRAFÍA}

\author{
NA ESCOLA, COM A ESCOLA INCORPORADA: PESQUISANDO O PROCESSO DE \\ SOCIALIZAÇÃO PESSOAL SOBRE O ACESSO À ESCOLA E O CORPO EM SALA \\ DE AULA POR MEIO DE UM EXERCÍCIO DE TRI-ETNOGRAFIA
}

IN SCHOOL, WITH SCHOOL WITHIN: EXPLORING PERSONAL SOCIALIZATION

AT SCHOOL AND THE BODY IN THE CLASSROOM THROUGH A TRIO-

ETHNOGRAPHY EXERCISE

Lucio Martinez-Alvarez*, Alfonso García-Monge*, Nicolás Julio Bores-Calle*
Palabras clave:

Etnografía.

Narrativas

personales.

Cuerpo.

Escuelas.

Palavras chave:

Etnografia.

Narrativas pessoais.

Corpo.

Escolas.

\begin{abstract}
Resumen: A través de un ejercicio de "trio-etnografía", tres docentes comparan la escuela a la que asistieron en su infancia con otra que observan en la actualidad y con lo que entienden como escuela ideal. En torno a dos situaciones de una jornada escolar (llegada a la escuela, en el aula) identificaron las diferentes presencias de lo corporal en la escolarización. Ese triple contraste entre memoria, presente e ideal permite reflexionar sobre la escolarización del cuerpo en un sistema que evoluciona y que, a su vez, los autores intentan superar. A partir de una narración etnográfica interpretativa, el objetivo de este texto es presentar al lector nuevos relatos personales que traten de temas como las marcas corporales de la ruptura con el entorno de protección familiar, los cuerpos escolarizados, los cuerpos silenciados, objetivados y rutinizados, los cuerpos expuestos, castigados 0 acosados, cuerpos seguros e inseguros.
\end{abstract}

Resumo: Por meio de um exercício de "tri-etnografia", três docentes comparam a escola que frequentaram na infância com uma observada atualmente e com o que entendem como escola ideal. Em torno de duas situações de uma jornada escolar (chegada na escola, em sala de aula) identificaram as diferentes presenças do corporal na escolarização. Este triplo contraste entre memória, presente e ideal permite refletir sobre a escolarização do corpo em um sistema que evolui e que, por sua vez, os autores procuram superar. A partir da narrativa etnográfica interpretativa, o objetivo deste texto é apresentar ao leitor novos relatos pessoais que tratem de temas como as marcas corporais da ruptura com 0 ambiente de proteção familiar; os corpos escolarizados; corpos silenciados, objetivados e rotinizados; corpos expostos, punidos ou assediados; corpos seguros e inseguros.

Abstract: By means of a 'trio-ethnography' exercise, three teachers compare the schools they attended with a current school and with their ideal school. Using two situations from a school day (entering the school; in the classroom), they identify situations and moments where the body is present in schooling. This triple contrast between memory, present and ideal allows them to understand ideas and reflections upon bodies' schooling within a changing system that, in turn, the authors attempt to overcome. Based on interpretive ethnographic narration, the text suggests new personal stories dealing with issues such as physical marks of the rupture with the family protection environment, schooled bodies, silenced, targeted and routinized bodies, exposed bodies, punished or harassed bodies, or confident and unconfident bodies.
*Universidad de Valladolid. Valladolid, España.

E-mail: lucio@mpc.uva.es

Recebido em: 11-12-2014

Aprovado em: 11-06-2015

(c) (1) (8) Licence 


\section{INTRODUCCIÓN Y OBJETIVO}

Para alguien que, como nosotros, pasó en las aulas buena parte de su niñez, luego estudió para ser profesor y, desde hace dos décadas, se dedica a formar docentes, nada hay más familiar que una escuela. Y quizá por eso, no dejamos de asombrarnos con los matices que descubrimos cada vez que nos acercamos a un colegio. A cada cambio nuestro ese "libro" que día a día se reescribe en cada una de las escuelas a las que tenemos acceso nos revela una parte de su complejidad, de sus aspectos comunes y de su diversidad. Y nos muestra, también, que quienes participamos de alguna manera en la construcción de esas escenas cotidianas reescribimos aquello de lo que somos testigos y protagonistas.

De manera sistemática, acudimos semanalmente a centros de Educación Primaria para compartir con niños y maestros un tiempo de su práctica escolar cotidiana. Después de años de esta presencia, en algunos centros, formamos ya parte de la dinámica escolar. Lo habitual en estos encuentros semanales es que estemos presentes y participemos en diferentes momentos con escolares. También solemos destinar un tiempo para dialogar con el maestro.

Mentiríamos si dijéramos que pasamos desapercibidos en las escuelas que visitamos. Somos como esa tía que viene a comer todos los jueves y que hace que ese día sea especial. Los alumnos esperan nuestra presencia como una de esas rupturas que tanto se aprecian en la rutina del día a día (no entraremos a discutir la ruptura de la privacidad de los niños tratada por HANAFIN; O'DONOGHUE; FLYNN; SHEVLIN, 2010; participamos de la idea de una escuela integrada y abierta a la comunidad).

El maestro también considera nuestra presencia algo especial, pese a que le implica algo más de exigencia autoimpuesta. Es el día que espera para poner a prueba alguna innovación o para comentar con un adulto temas variados que caracterizan un centro escolar; desde lo más educativo, a lo más relacional. Es, desde luego, muy especial para nosotros, profesores universitarios para quienes resulta complicado tener contextos escolares reales de los que nutrirnos y a partir de los cuales encarnar nuestras ideas y reflexiones.

Una de las estrategias que usamos para dinamizar y enriquecer la reflexión conjunta es elaborar relatos en los que resaltamos escenas que nos han llamado la atención por una u otra causa. Los relatos no tienen la pretensión de ser objetivos, pues somos conscientes de que todo texto retiene la impronta vital y profesional de quien lo selecciona, narra e interpreta (WOLCOTT, 2003).

Es como un juego de espejos, en el que la imagen se refleja en la superficie y es devuelta de nuevo, semejante pero modificada, al primer espejo. En este caso, los "espejos" nos muestran algunas maneras de ser de la práctica escolar, aunque tal vez lo que más claro queda al leerlos no es la práctica que se contempla, sino las personas que la miran, pues los relatos suelen revelar más de quienes los crean que de aquello sobre lo que tratan.

Por ello, el objetivo de este artículo es generar un relato para intentar que el lector o lectora se aproxime a sus recuerdos sobre las experiencias corporales de acceso al colegio, de ruptura con el entorno familiar y de estancia en las aulas, y los contraste con su situación e ideas actuales. 


\section{METODOLOGÍA}

Apoyándonos en la teoría de la estructuración (GIDDENS, 1995) para intentar comprendernos como sujetos socio-históricamente situados, pero agentes transformadores y reflexivos; partimos de una idea de indagación autoetnográfica, tomando el planteamiento de Breault, Hackler y Bradley $(2010,2012)$ para construir una "trio-ethnografia" en la que los autores indagan de forma dialógica en cómo sus experiencias vitales les han ido construyendo.

Esta metodología nos permitía explorar sobre nuestras propias narrativas a través del contraste con otros, llegando a significados emergentes y a textos abiertos con los que se pretende animar a los lectores a recordar y legitimar o cuestionar sus propias historias (NORRIS; SAWYER, 2012).

A través de un procedimiento dialógico los investigadores van tomando conciencia de sus construcciones, sus inconsistencias, sus preguntas sin resolver y los temas de fondo que soportan sus narrativas. Para que el contraste sea mayor y emerja más abundancia de significados el diálogo se establece no sólo entre tres personas, sino entre el presente y el pasado; entre el recuerdo y la vivencia actual; entre la realidad y el ideal; y entre la práctica y la teoría. Acceder a nuestras propias creencias resulta un reto difícil, pues necesitamos elementos para el "extrañamiento" que en este caso viene de los contrastes citados. ¿Cómo estructurar un procedimiento que permita establecer esos contrastes?

1a fase: Se filmaron 30 jornadas a lo largo de un curso con un grupo de escolares de 7-8 años de un colegio de una zona rural española.

$2^{2}$ fase: Posteriormente se organizaron los datos partiendo de algunos conceptos propuestos por diferentes autores:

- De Barbero (2007) en su estudio sobre los "capitales corporales en la escuela" los conceptos de "soltura", "torpeza", "cuerpo alienado", "sujeto-cuerpo" e "incorporeidad".

- De Kirk (2007) al hablar de "identidades incorporadas", las ideas de "escolarización", "institucionalización de prácticas", "regularización del cuerpo", "cuerpos escolarizados", "organización de espacios y tiempos escolares", "metas de docilidad-utilidad", "identidades incorporadas".

- De Moreno (2007) y su análisis sobre los "rituales corporales escolares" los ejes de: "organizaciones escolares", "actores", "disposiciones espaciales", "disposiciones temporales", "acciones simbólicas", "iconografía".

- De Vaca (2007) al mostrar las formas de estar del cuerpo en la jornada escolar los conceptos de "cuerpo silenciado", "cuerpo tolerado", "cuerpo libre", "cuerpo objeto de tratamiento".

Se utilizaron estos conceptos como punto de partida desde el que ordenar la estructura básica de análisis. Partimos de esos conceptos porque apreciábamos que resultaban válidos tanto para estructurar la mirada al pasado como para observar el presente. Sin embargo, a partir de ellos, se fueron añadiendo a la estructura analítica y luego narrativa otros conceptos y debates. 
3 a fase: A partir de un primer análisis de las filmaciones se narraron situaciones que representaban bien algunos temas fundamentales a la hora de definir las experiencias corporales en el proceso de escolarización.

4a fase: Cada uno de los autores construyó un relato personal en dos subfases:

Subfase 4.a.- Una primera con escritura libre centrada en las evocaciones sobre la escolarización que aparecían con más fuerza en el recuerdo. Cada autor realizó un relato personal rescatando imágenes, situaciones y sensaciones de su infancia. La pauta marcada en la construcción del relato personal era la de dejar que surgieran los momentos de mayor intensidad emotiva, sin limitaciones conceptuales para no quedarnos en estereotipos o vivencias demasiado construidas desde nuestros marcos teóricos actuales. No siempre conseguimos recuerdos nítidos, algunos fragmentos están encarnados a través de huellas que nos permiten revivir sensaciones (frío, miedo, ansiedad, catarsis, tedio...) más que situaciones claras. Por otra parte, nos encontramos con las limitaciones de escribir pensando en el juicio de los demás, ello lleva a una autocensura y a una "dulcificación" de ciertas vivencias para presentar una imagen más amable de nosotros mismos, aunque la discusión entre tres permite diluir la autoría personal en un relato de un "yo posible" aunque no necesariamente personal.

Subfase 4.b.-La segunda narración pretendía ser más sistemática, intentando hacer un repaso por aquellos temas que nos habían servido para hacer un primer análisis de las prácticas actuales filmadas. Se trataba de contrastar las vivencias de los escolares del colegio observado con nuestro recuerdo sobre nuestra escolarización. En la elaboración de estas narraciones se ponía de manifiesto el intrincado juego entre estructura y agencia. Veíamos que al acceder a nuestros recuerdos lo hacíamos desde nuestros marcos teóricos actuales. Entendíamos que esos conceptos funcionaron un poco a modo de "attractors" (PRIGOGINE, 1980), como forma de dotar de cierto orden a la experiencia pasada y presente (SKARDA; FREEMAN, 1987). Pero, a la vez, queríamos rescatar relatos más cercanos a la experiencia vivida. Esta es una limitación y característica de este tipo de trabajos, el pasado se re-construye selectivamente y como señala Toren (1994: 979) hay una incapacidad de evocación discursiva de la experiencia que generó el rastro, no hay un "acceso transparente" al pasado. Como dijera Heráclito, uno no se baña dos veces en el mismo río, y cuando lo hace, uno ya no es tampoco el mismo, a modo del "ratchet effect" (TOMASELLO, 1999).

5 a fase: En la siguiente fase, cada autor presentó su narración e interrogó a los otros para clarificar y matizar más aspectos de las experiencias. Este diálogo y contraste es uno de los puntos críticos de esta metodología pues permite clarificar situaciones, indagar en construcciones y desentrañar significados.

Con esos nuevos relatos más detallados y justificados se realizó un triple trabajo:

- Una puesta en común sobre los aspectos que compartían las tres experiencias (Justapositon of experiences).

- Una labor de análisis de las creencias implícitas sobre cada tema y la estructuración sufrida a lo largo de la escolarización personal.

- Un contraste entre las ideas que habíamos normalizado sobre la educación a lo largo de nuestra escolarización y nuestras ideas actuales, rescatando aquellos documentos 0 experiencias formativas que nos habían ayudado a hacer este cambio, a vislumbrar otras 
formas de ver y entender las experiencias, y a poner de manifiesto las contradicciones y debates que no logramos resolver.

6므 fase: Finalmente se realizó una selección de fragmentos de práctica educativa actual a los que se ligaron fragmentos de las narraciones de la experiencia de los autores y comentarios que permitieran contrastar las experiencias con nuestras ideas actuales para crear un texto único y compartido y escrito en primera persona del singular como en primera del plural en múltiples idas y venidas temporales. El resultado ha sido un escrito intencionalmente poliédrico y polifónico, que combina múltiples niveles de reflexión sobre la práctica y múltiples perspectivas.

El relato final se articulaba en torno a cuatro momentos habituales en una jornada escolar: el tránsito del cuerpo hacia la escuela, el cuerpo en el aula, el cuerpo en los recreos, y el cuerpo en la clase de educación física. En este artículo presentaremos los dos primeros.

Las referencias bibliográficas se han añadido como elemento de contraste con la narración, con la intención de reflejar los cambios de sensibilidad y enfoque que la comunidad profesional ha ido sufriendo sobre diferentes temas tratados, además de mostrar algunas de las influencias que han ido construyendo y modificando nuestras perspectivas. Para que el relato no pierda coherencia narrativa se ha optado por sacar estas referencias fuera del cuerpo central del texto. La intención con las referencias bibliográficas es aportar una especie de paisaje teórico en el que estamos inmersos. No usamos muchos de esos conceptos tanto con fines analíticos sino como ventanas que muestren más aspectos de nuestra construcción y den pistas de contraste a un posible lector que quiera interrogarse sobre su propio proceso; porque realmente el eje de la construcción del artículo pretende que sea, como señalan Norris y Sawyer (2012, p.10), explorar y articular narrativas personales y colectivas, usando a uno mismo como lugar de indagación sobre la socialización y la inscripción cultural, estableciendo un diálogo entre el texto y el lector, problematizando la alineación del lector con metanarrativas implícitas.

\section{COMO ARROYOS QUE CONFLUYEN EN EL VALLE}

La mañana amaga con lluvia y viento, sin decidirse a ser del todo invernal. Quedan quince minutos para que suene el timbre y el vestíbulo estaría vacío si no fuera por un niño de 3 años que espera, tímido, junto a la cuidadora. Frente a la puerta de entrada, aparca uno de los autobuses que transporta escolares de los pueblos colindantes. Los más mayores bajan los primeros y se acercan corriendo a la cuidadora para pedirle que hoy les deje quedarse jugando en el patio hasta que suene el timbre, pero ella les responde que saben que deben quedarse dentro del edificio."Al colegio se viene a estudiar y no a jugar", añade.

Ahora hay más movimiento. Llegan autobuses de otras rutas y también los niños del pueblo. Alguno de los más mayores viene andando desde casa; otros, llegan en coche, acompañados por sus padres hasta la verja del colegio. Los hay que cruzan el patio parsimoniosamente; otros a la carrera, sobre todo cuando ven que las filas ya están hechas y ellos son los últimos.

Los profesores aguardan en el porche, donde los alumnos hacen las filas por cursos. Se nota a los profesores de los cursos inferiores más pendientes de sus alumnos, pues se colocan pronto al frente de sus filas. Los de los mayores están más relajados y continúan un rato hablando en grupo. 
Recuerdo cientos de mañanas caminando hacia el colegio, cada una diferente, todas iguales. No olvido el frío que sentía nada más salir a la calle, ni la sensación en el estómago los días de algún examen difícil o cuando había olvidado hacer los deberes. También recuerdo la angustia de decidir cada mañana si tomar el atajo del "callejón de la fuente", que me evitaba dar un buen rodeo, pero que acarreaba siempre el riesgo de encontrarme con que el perro del taller estuviera suelto ${ }^{1}$.

En los años setenta, los escolares españoles íbamos solos al colegio desde los seis 0 siete años. Yo salía del portal y recorría con la mirada toda mi calle para ver si divisaba alguien con quien ir hasta el colegio, aunque no fuera de mi clase. Mi madre me seguía con la mirada desde su ventana. Un momento antes de doblar la esquina, yo me volvía para despedirme. Sólo entonces giraba ella su cuerpo y se iba a retomar las tareas de casa. En la ventana quedaba, durante unos segundos, el vaho que su mano cálida había dejado en el frío cristal.

A fuerza de repetir este ritual, abandonar el hogar y acudir al colegio dejó de ser un acontecimiento. Cuando el perro del taller se fue haciendo viejo y yo más mayor, dejé de tenerle miedo. También fui olvidándome poco a poco de despedirme desde la esquina, más atento a la alegría del reencuentro con compañeros con los que tramar todo tipo de planes que no permitían demora. Sólo años después caí en la cuenta de que la mano de mi madre habría permanecido allí muchos días mientras yo me dirigía, con más o menos ganas, hacia el colegio, plenamente asumido que ése era el escenario donde había de pasar las mañanas y las tardes de los días lectivos.

La transición del hogar a la escuela no resulta sencilla tampoco para los escolares actuales. Al igual que tuve que hacer yo, los niños de hoy en día tienen que luchar contra sus miedos particulares al abandonar la seguridad del hogar en una ruptura que se produce cada vez a edades más tempranas ${ }^{2}$.

Actualmente casi ningún niño viene solo desde casa, pero apenas hay algún padre que entra al centro. La verja de entrada parece ser una frontera que marca el límite donde se ejerce el cambio de custodia de los pequeños entre su familia y la escuela. El patio es una tierra de nadie, cruzada de manera presurosa para incorporarse a las filas y entrar luego a los edificios. Tal vez el juego pudiera ofrecer una transición más amable, pero a veces da la sensación de que no tiene cabida más que en los márgenes de la escuela y puede, incluso, llegar a ser considerado un riesgo añadido que los educadores no quieren asumir ${ }^{3}$.

El timbre recuerda a una fábrica que reclama hasta el último minuto del horario laboral. El resto es considerado como tiempo perdido o como algo de lo que la escuela se desentiende, pues no es su cometido. Tal vez por eso los niños mayores de la escena narrada intentan apurar el tiempo de juego antes de que suene el timbre, como da el buceador una última bocanada de aire antes de sumergirse.

\footnotetext{
1 Muchos han sido los trabajos que nos han mostrado la importancia de estos tiempos en los que se produce la discontinuidad (SPINDLER, 2005) en la vida de los niños y niñas y cómo estos van creando su yo-cuerpo en el espacio, van entendiendo el entorno y estableciendo relaciones afectivas con el espacio (HART, 1979; PROSHANSKY, FABIAN, KAMINOFF, 1983; VAN MANEN, 1990).

2 En la actualidad hay una preocupación creciente por la seguridad de los niños en esos tiempos de transición del hogar al colegio. Ya sea por cuestiones de seguridad vial, inseguridad ciudadana o preocupación por el sedentarismo, se intentan promover rutas seguras y estilos de vida activos (KEARNS; COLLINS, 2003; MCDONALD; AALBORG, 2009; ZHOU; YANG; HSU; CHEN, 2010) a la vez que la Sociedad intenta poner remedio a formas de vida consideradas "insostenibles" (KYTTÄ, 2004; CHATTERJEE, 2006). Intervenciones de control social que nos remiten al concepto denunciado por Bernstein de "totally pedagogised society", estudiado por Evans, Rich, Davies y Allwood (2005).

3 La mirada sospechosa hacia el juego por parte de la institución escolar es antigua. Ya el humanista Luis Vives la denunciaba en 1535 (1998). Desde el siglo XVI encontramos muchas prohibiciones para que el juego no traspasara los límites de la institución escolar (BAJO; BELTRÁN, 1998).
} 
La transición del hogar a la pequeña sociedad que se forma en cada clase y cada escuela nos lleva de un entorno en el que somos el centro a otro en el que hay que acomodarse a otros ritmos y en los que hay que compartir atenciones y cuidados con otros niños y niñas. Esta separación forzosa de nuestro entorno familiar nos fuerza a cambiar y valernos por nosotros mismos. También, a plantearnos quiénes somos, quiénes nos dejan ser y quiénes queremos ser. Pero no estamos seguros de que se aproveche la oportunidad de construir una comunidad que no desgaja a las niñas y los niños del hogar del que provienen, sino que lo amplía y enriquece, gracias al resto de escolares de diferentes edades y junto con los adultos que velan por ellos. La forma más habitual en la que vemos que se organizan las escuelas es en torno a las clases homogéneas, en horarios estrictos marcados por los timbrazos que señalan el inicio y el final de cada periodo. Quedan fuera de la atención pedagógica los momentos "periféricos" y los grupos mezclados, siempre difíciles de encasillar y de encauzar.

¿Qué necesitarían los docentes para entender la experiencia profunda, no ya sólo académica, que supone para los pequeños entrar en la escuela? ¿qué les hemos dicho en la universidad? ¿qué sabemos sus formadores? ¿qué modelos alternativos les ofrecen nuestras propias asignaturas, también circunscritas, por lo general, al momento entre los timbrazos (en este caso simbólicos, pero no menos reales) de entrada y de salida que dan por supuesto que el resto de la vida de nuestros estudiantes se coloca entre paréntesis?

Nosotros colaboramos con la construcción de Comunidades de Aprendizaje ${ }^{4}$, abriendo la escuela a la sociedad e integrándola en la comunidad que le da sentido. Es complicado, lo sabemos. No debe ser fácil transformar esta manera de ver las cosas, pues esta escena, con mil matices, la vemos repetida en otros muchos colegios a los que acudimos. Existe una cierta comodidad en esta división tajante entre lo escolar -entendido como académico- y lo extraescolar. Es un modelo que pide al niño que mientras dure la jornada escolar deje entre paréntesis su vida, como colgada del perchero de la entrada. Pero este aislamiento generalizado no sólo se da con el entorno más inmediato, sino que está presente también dentro de los centros, pues comprobamos con frecuencia que los escolares de una clase no conocen ni el nombre de sus compañeros de la clase de al lado.

\section{CUERPOS CELESTES, CUERPOS TERRENALES}

A primera hora, los de Segundo (7-8 años) tienen Conocimiento del Medio, "Cono", como ellos dicen. Entran en el aula-gimnasio en la que Pablo da todas sus clases, y éste les pide que preparen el libro y el cuaderno de tareas. Algunos lo hacen rápidamente, otros se quedan con la mirada perdida en algún punto, la mayoría se mueve con lentitud. El tono de jugueteo que traían por el pasillo ha cambiado por otro de suplicio.

El profesor les pide que lean el capítulo del libro donde se habla sobre la Luna y las estrellas. Juan y Jaime leen el texto concentrados; Arancha se remueve sin leer y juega con su estuche; Raquel se hace la remolona, pero acaba poniéndose a la tarea; Lidia habla con todos un poquito; un pupitre vacío refleja que María ha faltado otra vez...

Ahora pide a alguien que empiece a leer en alto el texto y, luego, va pidiendo a otros que sigan. Mientras están leyendo, Lidia no puede parar de moverse: se

4 Para entender la idea de "Comunidades de Aprendizaje" a la que nos referimos se puede ver el trabajo de Elboj, Puigdellívol, Soler y Valls (2009). 
sienta, se estira, bosteza. A Arancha, que está sentada a cierta distancia de sus compañeros, le cuesta centrarse.

Pablo hace un alto en la lectura para preguntar si saben cómo se mueve la Tierra respecto al Sol. De pronto, como si súbitamente hubiera recordado algo, sale de la clase. Vuelve al poco con un modelo planetario fabricado, con pelotas y alambres, que representa el Sol, la Luna y la Tierra. Se sienta entre los alumnos mientras les muestra cómo se mueven los astros. Juan mira fijamente al modelo y dice que está hecho con pelotas. "¿La Tierra gira en torno al Sol?", pregunta el profesor. "El Sol”, repite como un eco Arancha.

Raquel comenta que la Tierra no puede ir por debajo del Sol y el profesor les pregunta si la Tierra se mueve al lado del Sol, o por arriba y por debajo de él, o cómo. Pablo va a buscar un globo terráqueo y trata de explicarles que no hay arriba ni abajo. Les cuesta entender el tema, pero los alumnos han cambiado de actitud. Es como si hubieran despertado. Han ido desapareciendo los bostezos y otros síntomas de aburrimiento y desinterés. Escuchan con una sonrisa en sus caras. De vez en cuando, responden a alguna de las preguntas que Pablo les hace. Están absortos contemplando el modelo y las explicaciones de Pablo. Algunos se ponen de pie para ver más de cerca el modelo. El profesor se lo deja y muchos lo manipulan interesados.

Sara comenta que el Sol es la estrella más grande. El profesor les muestra un vídeo en el ordenador en el que aparecen los tamaños relativos de planetas y estrellas. Se quedan asombrados.

Para acabar la clase de "Cono", el profesor les propone un juego con una "trampa" que deben descubrir. Les coloca a todos en medio del gimnasio, en un círculo mirando hacia fuera. Les dice que ellos representan la Tierra. El profesor lleva en la mano un balón amarillo que representa el Sol y da vueltas en torno al corro, haciéndoles ver cómo se producen el día y la noche. Les pregunta que cuál es la trampa, y Sara responde que el Sol va demasiado deprisa. Raquel, casi al mismo tiempo, dice que no gira el Sol, sino la Tierra. Después, el profesor pide a dos niños que representen los movimientos de rotación y translación de la Tierra. El resto se ríe y aplaude la representación. Pablo les dice que ahora lo deben hacer todos por parejas. Lo hacen bastante bien y con mucha implicación.

Cuando acaban, Pablo les anuncia que van a empezar con "Lengua", que cambien los libros y que quien quiera vaya al baño ahora, porque después ya no se podrá. Se van todos al baño.

No logro comprender muy bien por qué nos peleábamos para colocarnos los primeros de la fila, pero recuerdo la sensación de malestar mientras subía las escaleras, así como la tensión durante el rezo de la mañana, seguido por la interminable letanía de pasar lista a los 45 alumnos. Todavía me sé algunos trozos de la lista, nombres y apellidos, de corrido. Mezclo en mi recuerdo las aulas y los cursos, porque la impresión siempre era la misma: una nueva mañana por delante sin poder moverme del pupitre. La mera perspectiva era una pesadilla, aunque tenía muchas maneras de evadirme de aquella realidad: colocaba los lapiceros como si fueran vagones de tren, jugaba con las canicas que tenía en el bolsillo, pintarrajeteaba el pupitre y luego lo emborronaba al querer limpiarlo frotándolo con un poco de saliva, disparaba bolas de papel con una improvisada cerbatana hecha con la caña de mi bolígrafo ${ }^{5}$...

Hace ya unos minutos que la voz del profesor de Ciencias Naturales se ha convertido en un murmullo y que mi atención está fijada en el calendario que cuelga al lado de la pizarra: 
17 de noviembre de 1978. Me entretengo en calcular que cuando llegue el año 2000 seré tan viejo como el profesor. Súbitamente, siento las miradas de todos mis compañeros y me pongo rojo como un tomate. El profesor está diciendo mi nombre y la clase ha estallado en carcajadas forzadas y en golpes descontrolados a las mesas. A pesar del aturdimiento, comprendo con lucidez que esta tarde tendré que quedarme castigado ${ }^{6}$.

Las interacciones predominantes entre los profesores y alumnos en mi escuela eran de desapego y frialdad. La distancia iba más allá de las formas de trato, de los castigos o de las humillaciones en la pizarra ante el resto del grupo. También era una distancia hacia la ciencia, el arte 0 las humanidades ${ }^{7}$.

Salir airoso de una batalla no era garantía de lo que podría ocurrir en el próximo embate. El conocimiento era algo que podía volverse contra ti, un enemigo que traía represalias crueles. El profesor te mandaba acercarte a su tarima y colocarte firmes para recibir el castigo si no habías sido capaz de dar la respuesta exacta. Otras veces, el profesor te colocaba en situaciones de máxima tensión exponiéndote ante el resto; tanto daba si era para recibir un halago no deseado o para que los demás se burlaran de tu estupidez. Lejos de compadecernos en nuestra desventura mutua, la tensión era tan alta que celebrábamos no ser el objeto de escarnio burlándonos del pobre desgraciado de turno. Cruel sistema aquel que lleva a los miserables a escarnecerse entre ellos.

En ese entorno, cualquier ruptura de la normalidad se convertía en motivo de jolgorio; la entrada de alguien en clase, un ruido inusual en la calle, la caída de una silla... A veces, un profesor se atrevía a traernos un material nuevo -tal vez un mapa- o quizá nos llevaba al laboratorio a realizar un experimento, pero nuestra necesidad de desahogo era tan grande que la iniciativa acababa muchas veces en alboroto y en el arrepentimiento del docente por haberse salido del guión.

La clase que Pablo ha impartido hoy está muy alejada de esos recuerdos. Muestra un interés por la materia y por el aprendizaje que logra vencer las reticencias iniciales de los estudiantes hacia las partes más áridas y consigue algo que apenas se daba en nuestra infancia: que los contenidos sean vistos no sólo como algo que hay que memorizar, sino como algo en cuya construcción se puede colaborar ${ }^{8}$.

Desde la perspectiva de un educador físico, hay otro aspecto en el que esta clase contrasta con lo que era habitual en nuestra infancia. En gran parte del horario escolar parecía aspirarse a que los niños fuéramos sólo cabeza o, mejor aún, sólo mente9 .

Junto a formas más tradicionales en las que el libro de texto conducía el aprendizaje de forma homogénea y aislada de los compañeros, hemos visto en esta escena otros momentos en los que los niños aprendían con todo el cuerpo y en grupo; los textos abstractos cobraban vida cuando se hicieron tangibles y cuando el alumnado se implicó emocional y socialmente

\footnotetext{
6 Más allá de comprender la disciplina como un conjunto de herramientas utilizadas para establecer un control y moldeamiento de personas útiles y dóciles (FOUCAULT, 1995) nos interesan trabajos como los de Depalma, Membiela y Pazos (2011) que muestran cómo los propios docentes intentan dar alternativas a lo que vivieron en su escolarización.

7 Para comprender la organización de las escuelas y las relaciones profesorado-alumnado basadas en una fuerte ideología de religión, orden, vigilancia, disciplina, jerarquía, competición y escasa formación de solidaridades nos ayuda la obra de Markus (1996), aunque aspiramos a modelos superadores de estas dinámicas como los expuestos por Elboj et al. (2009).

8 Nos resulta de gran inspiración para entender la relación pedagógica y el "tacto pedagógico" la obra de Merieu (1992) y de Van Manen (1991) 9 Una crítica al desdoble cuerpo-mente la encontramos en Shapiro (1994). En la construcción de nuestra visión sobre el cuerpo en la escuela han tenido gran influencia la obra de Francesco Tonucci (2007), la de Loris Malaguzzi (HALL et al., 2010), así como las propuestas de Hildebrandt-Stramann (2005) y de Vaca (2007), mostrándonos otros modelos escolares que intentan combatir el dualismo cuerpo-mente.
} 
en aprender. El escenario de la clase muestra el deseo de traspasar las fronteras en las que con tanta frecuencia se encierra la escolaridad. El propio hecho de que se trate de un gimnasio invita al movimiento, a abandonar, aunque sea temporalmente, el pupitre para que haya una mayor cercanía con el profesor y los coaprendices, o para experimentar en carne propia los conceptos tratados.

Este fragmento nos revela así mismo que el aprendizaje de los contenidos se construye en un entorno social que le da sentido y que lo impulsa en una u otra dirección. Aprendemos junto a otros y en interacción con otros. Esto funciona para lo bueno y para lo malo. Lo mismo que el grupo puede empujar y elevar, puede arrastrar y hundir.

El deseo de aprender reside, en gran medida, en estar a gusto con otros y con uno mismo. En no sentirnos ridículos si fallamos. En atrevernos a ir más allá de los lugares que nos resultan comunes, porque tenemos confianza en que estamos en un entorno seguro. En sentir que el nuevo aprendizaje es algo que nos une a aquellos con quienes lo hemos logrado. El grupo puede así construir, no sin esfuerzo y no sin la ayuda del adulto, lo que Philipe Meirieu denomina un "ambiente libre de amenazas".

Pero también encontramos entornos letales para el aprendizaje, que parecen poner a los estudiantes en guardia 0 , directamente, al ataque. Lugares donde el conocimiento es un arma que los estudiantes se arrojan entre sí. Lugares en donde la menor de las preocupaciones es apropiarse del contenido y crecer gracias a ello, pues están en juego cosas más importantes como la autoestima, la aceptación de los iguales o la supervivencia en un entorno hostil hacia el conocimiento.

\section{CONSIDERACIONES FINALES}

En diálogo constante con el entorno, vamos co-construyéndonos en un flujo incesante de experiencias y creación de nuevos significados. Lo que un día dimos por natural, en nuestro proceso de socialización se fue cuestionando y generando nuevos ideales y prácticas.

Las experiencias más desagradables, en contraste con nuevos marcos teóricos a los que fuimos abriéndonos, lejos de ser un lastre encorsetador, nos ayudaron a ambicionar otras formas de atender a la persona.

Las huellas corporales de nuestras malas experiencias nos sirven como alarmas sensibilizadoras para comprender las vivencias de nuestro alumnado y las experiencias por las que no queremos que pasen.

Son muchos los dilemas a los que seguimos sin dar respuesta y son muchos los nuevos enfoques, discursos y sensibilidades que aparecen ante lo que resultaba cotidiano. Asumimos esta provisionalidad, pero, a la vez, intentamos dotarnos de valores más sólidos (respeto e inclusión, integración de la globalidad de la persona o permeabilidad del los centros escolares al entorno social) sobre los que asentar nuestras propuestas.

\section{REFERENCIAS}


BARBERO GONZÁLEZ, José Ignacio. Capital(es) corporal(es) que configuran las corrientes y/o contenidos de la Educación Física Escolar. Agora para la Educación Física y el Deporte, Valladolid, n. 4, p. 21-38, 2007.

BREAULT, Rick; HACKLER, Raine; BRADLEY, Rebecca. Constructing a male teaching identity: A trio-ethnography. En: CONGRESS OF QUALITATIVE INQUIRY,6., 2010. Disponible en: $<$ http://www.breaultresearch.info/trio-ethnography.html >. Acceso en: 10 mar. 2011.

BREAULT, Rick; HACKLER, Raine; BRADLEY, Rebecca. Seeking Rigor in the Search for Identity: A Trioethnography. En: NORRIS, J.; SAWYER, R.D.; LUND, D. Duoethnography: Dialogic methods for social, health, and educational research. Walnut Creek, CA: Left Coast, 2012. p. 115-136.

CHATTERJEE, Sudeshna. Children's friendship with place: an exploration of environmental child friendliness of children's environments in cities. 2006. Dissertation (Doctoral). North Carolina State University, 2006.

DEPALMA, Renée; MEMBIELA, Pedro; SUÁREZ, Mercedes. Teachers' memories of disciplinary control strategies from their own school days. British Journal of Sociology of Education, London, v.32, n.1, p.75-91, ene. 2011.

ELBOJ SASO, Carmen; PUIGDELLÍVOL AGUADÉ, Ignasi; SOLER GALLART, Marta; VALLS CAROL, Rosa. Comunidades de aprendizaje: transformar la educación. Barcelona: Grao, 2009.

EVANS, John; RICH, Emma DAVIES, Bryan; ALLWOOD, Rachel The Embodiment of Learning: what the sociology of education doesn't say about 'risk' in going to school. International Studies in Sociology of Education, Wallingford, v.15, n.2, p.129-148, 2005.

EVERHART, Robert B. Leer, escribir y resistir. En: VELASCO, Honorio.; GARCÍA, Francisco J.; DÍAZ DE RADA, Ángel. Lecturas de antropología para educadores. Madrid: Trotta, 2005. p.205-241.

FOUCAULT, Michel. Discipline and Punish: the Birth of the Prison. New York: Vintage, 1995.

GIDDENS, Anthony. La constitución de la sociedad. Bases para una teoría de la estructuración. Buenos Aires: Amorrortu, 1995.

HALL, Kathy; HORGAN, Mary; RIDGWAY, Anna; MURPHY, Rosaleen; CUNNEEN, Maura; CUNNIGHAM, Denice. Loris Malaguzzi and the Reggio Emilia experience. London: Continuum International, 2010.

HANAFIN, Joan; O'DONOGHUE, Tom; FLYNN, Marie; SHEVLIN, Michael The primary school's invasion of the privacy of the child: unmasking the potential of some current practices.

Educational Studies, Mahwah, v.36, n.2, p.143-152, 2010.

HART, Roger. Children's experience of place. New York: Irvington, 1979.

HILDEBRANDT-STRAMANN, Reiner. Escola (s)em movimento. Movimento, Porto Alegre, v.11, n.1, p. 121-139, jan./abr. 2005.

KEARNS, Robin A.; COLLINS, Damian C.A. Crossing roads, crossing boundaries: empowerment and participation in a child pedestrian safety initiative. Space and Polity, Abingdon, v. 7, n.2, p.193-212, 2003. 
KIRK, David. Con la escuela en el cuerpo, cuerpos escolarizados: la construcción de identidades inter/nacionales en la sociedad post-disciplinaria. Agora para la Educación Física y el Deporte, Valladolid, v. 4, p. 39-56, 2007.

KYTTÄ, Marketta. The extent of children's independent mobility and the number of actualized affordances as criteria for child-friendly environments. Journal of Environmental Psychology, London, v.24, n.2, p. 179-198, jun. 2004.

MARKUS, Thomas A. Early Nineteenth Century School Space and Ideology. Paedagogica Historica, Gent, v.32, n.1, p.8-50, 1996.

MCDONALD, Noreen. C.; AALBORG, Annette E. Why Parents Drive Children to School: Implications for Safe Routes to School Programs. Journal of the American Planning Association, Chicago, v.75, n.3, p.331-342, 2009.

MEIRIEU, Philipe. Aprender sí, pero ¿cómo?. Barcelona: Octaedro, 1992.

MORENO GÓMEZ, William. La educación corporal en los rituales escolares. Agora para la Educación Física y el Deporte, Valladolid, n.4, p.11-129, 2007.

NORRIS, Joe; SAWYER, Richard D. Toward a Dialogic Methodology. En:NORRIS, J.; SAWYER, R.D.; LUND, D. Duoethnography. Dialogic Methods for Social, Health, and Educational Research. Walnut Creek: Left Coast, 2012. p. 9-39.

PRIGOGINE, llya. From Being to Becoming: time and complexitiy in the physical sciences. San Francisco: W.H.Freeman. 1980.

PROSHANSKY, Harold. M.; FABIAN, Abbe. K.; KAMINOFF, Robert. Place-identity: physical world socialization of the self. Journal of Environmental Psychology, London, v.3, n.1, p.5783, Mar. 1983.

SHAPIRO, Sherry. Re-membering the body in critical pedagogy. Education \& Society, Melbourne, v.12, n.1, p.61-79, 1994.

SKARDA, Christine A.; FREEMAN, Walter J. How Brains make chaos in order to make sense of the World. Behavioural and Brain Sciences, Cambridge, v.10, n.2, p.161-195, 1987.

SPINDLER, George D. La transmisión de la Cultura. En: VELASCO, Honorio; GARCÍA, Francisco J.; DÍAZ DE RADA, Ángel. Lecturas de antropología para educadores. Madrid: Trotta, 2005. p. 205-241.

TOMASELLO, Michael. The Cultural Origins of Human Cognition. Cambridge: Harvard University, 1999.

TONUCCI, Francesco. Frato, 40 años con ojos de niño. Barcelona: Grao, 2007.

TOREN, Christina. Mind, Materiality and History: Explorations in Fijian Ethnography. London: Routledge, 1994.

VACA ESCRIBANO, Marcelino J. Un proyecto para una escuela con cuerpo y en movimiento. Ágora para la Educación Física y el Deporte, Valladolid, n. 4, p. 91-110, 2007.

VAN MANEN, Max. Researching lived experience: Human science for an action sensitive pedagogy. Albany: SUNY, 1990. 
VAN MANEN, Max. The tact of teaching. Albany: SUNY, 1991.

VIÑAO, Antonio. Tiempos escolares, tiempos sociales. Barcelona: Ariel, 1998.

VIVES, Luis. Diálogos sobre la educación. Barcelona: Altaya, 1998.

WOLCOTT, Harry F. Mejorar la escritura de la investigación cualitativa. Medellín: Universidad de Antioquia, 2003.

ZHOU, Huaguo; YANG, Jidong; HSU, Peter; CHEN, Shaoqiang. Factors Affecting Students' Walking/Biking Rates: Initial Findings from a Safe Route to School Survey in Florida. Journal of Transportation Safety \& Security, New York, v. 2, n.1, p.14-27, 2010. 